\title{
Dominance of toroidal oscillations in dawn/dusk sectors: A consequence of solar wind pressure variation
}

\author{
A. K. Sinha and R. Rajaram
}

Indian Institute of Geomagnetism, Colaba, Mumbai 400 005, India

(Received June 22, 2001; Revised January 14, 2003; Accepted February 6, 2003)

\begin{abstract}
The pressure variations in the solar wind produce the oscillations in surface currents at the magnetopause boundary in order to nullify the pressure imbalance. These currents introduce compressional variations in the magnetic field within the magnetosphere. The response of transverse field line oscillations to such changes in the magnetic field has been brought out in perfectly reflecting ionospheric conditions. The analysis clearly shows that the fundamental toroidal modes are dominant in the dawn and the dusk sectors as revealed by the statistical studies of pulsations observed by the satellite AMPTE/CCE (Anderson et al., 1990). It is traditionally believed that such oscillations are mainly driven by Kelvin-Helmholtz $(\mathrm{K}-\mathrm{H})$ instability (Anderson et al., 1990). Our analysis shows that the dominance of fundamental toroidal modes in the dawn and dusk sectors can also be explained in terms of response to impressed pressure impulses without invoking $\mathrm{K}-\mathrm{H}$ instability. The analysis also shows that poloidal modes do not exhibit any longitudinal structures. These results are consistent with the observations (Anderson et al., 1990).
\end{abstract}

\section{Introduction}

Field line oscillations along select field lines are known to respond to the sudden change in solar wind pressure variation (Baumjohann et al., 1984). To locate the site of dominant response and obtain at least a qualitative picture of the magnitude and location of these oscillations, a working model is required for the impulse delivered at any given point within the magnetosphere. Changes in solar wind dynamic pressure result in the re-adjustment at the magnetopause boundary and the corresponding surface currents required for pressure balance. In the first order of approximation, estimates of the field can be obtained by ignoring the plasma currents within the magnetosphere and computing the impulse magnetic field due to surface currents at the magnetopause boundary. In that case if we express the magnetopause current in the same manner as Mead (1964) treated the problem, we will obtain distribution identical to what Mead obtained for magnetic field within the magnetosphere. This will be an oversimplification as the radial dependence due to the propagation characteristics of the compressible mode cannot be included. The model (Mead, 1964) provides the net field due to these surface currents for a given magnetopause stand-off distance and has been used effectively to estimate the magnetic field associated with storm time sudden commencement and sudden impulse (cf. Schulz and Lanzerotti, 1974) to understand the role of these transient variation in cross field diffusion and drift echoes. The model should also provide a working system for a preliminary understanding of the response of transverse field line oscillations by solar wind pressure variation.

Copy right(C) The Society of Geomagnetism and Earth, Planetary and Space Sciences (SGEPSS); The Seismological Society of Japan; The Volcanological Society of Japan; The Geodetic Society of Japan; The Japanese Society for Planetary Sciences.
We do not undermine the importance of propagation effects. Lee (1996) numerically studied the dynamics of the wave propagation penetrating the plasmapause in a dipole field geometry. Though in this paper Alfvén velocity was assumed to vary only radially, the paper could demonstrate that the MHD waves can propagate deep into the plasmosphere through tunneling and could excite field line oscillations. Further work was done by Itonaga et al. (1997) to simulate the transient response of the magnetosphere to the step-like and impulse-like disturbances in dipole field geometry by taking altitude distribution of Alfvén velocity. They could show that the magnetospheric response to such disturbances is one-dimensional cavity-mode type that could be one of the cause of equatorial $\mathrm{Pi} 2$ pulsations. Fujita et al. (2000), in order to explain the Pi2 pulsations numerically studied the transient impulse response in dipole field geometry taking source current that modeled the substorm current wedge. The very recent work Fujita et al. (2001) investigated the propagation property of transient MHD waves based on realistic magnetosphere-ionosphere model. Their results suggest that the field line resonance in the plasmosphere is almost simultaneously excited with the passing of the wave front of the initial impulse. By assuming instant propagation we do not include these important effects. These may be incorporated in future studies within the same model scenario.

The existence of field line resonances in the Earth's magnetosphere is a well established phenomena backed up by theory and observations (Dungey, 1954; Sugiura, 1961; Nagata et al., 1963; Cummings et al., 1969; Singer et al., 1981; Anderson et al., 1990; Sinha and Rajaram, 1997 and references therein). Though in real magnetospheric conditions, coupled modes involving moderate axial asymmetry in the 
wave fields are likely, for analytic simplicity we confine ourselves to the so-called 'toroidal' and 'guided poloidal' fieldline resonance mode. The axisymmetric toroidal mode is a transverse Alfvén mode with torsional oscillations of the entire magnetic shell while the poloidal mode corresponds to the oscillations of the magnetic field lines in the meridional plane. Though it has been pointed out that (Anderson et al., 1990) poloidal mode is not excited well enough and hence not frequently observed whereas toroidal oscillations are long-lived phenomena and are observed very frequently, poloidal oscillations are well documented magnetospheric phenomena (Cummings et al., 1969; Southwood and Hughes, 1983). These two modes are decoupled solutions of the coupled hydromagnetic wave equations and the decoupling is affected by assuming the axisymmetry as in case of toroidal or high asymmetry as in case of guided poloidal modes. These modes have natural frequencies arising through perfect reflection at the conjugate ionospheres. Perfect reflection at the ionosphere can be achieved in either of the two extreme conditions in the ionosphere i.e. (a) The ionospheric conductivity is infinitely large, and (b) The ionospheric conductivity is vanishingly small (Southwood and Hughes, 1983). In case of the ionosphere being infinitely conducting, the electric field and field line displacement are very small at the ionosphere and hence is called as 'rigidend' mode. Whereas, in the other extreme when the ionospheric conductivity is very small, the standing mode with very small magnetic field fluctuation but large electric field and field line displacements at the ionosphere takes place and hence is termed as 'free-end' mode. A very low conductivity of the ionosphere is the typical nighttime condition and gives rise to the situation where the magnetic fields of the incident and reflected waves tend to cancel, whereas the electric field and displacement of the foot of the field line in the ionosphere are large. This results in the standing modes with a free-ended boundary condition for the end of the field line (Newton et al., 1978).

The general characteristics of such modes have been studied for special cases by various authors (Allan et al., 1985, 1986; Southwood and Kivelson, 1990). Whereas Allan et al. $(1985,1986)$ assumed the cylindrical geometry, Southwood and Kivelson (1990) worked with Cartesian box model. Fujita and Patel (1992) through numerical simulation studied the eigenmodes of coupled global oscillations for finite azimuthal wave number in dipole field geometry for infinitely conducting ionospheric conditions. They clearly demonstrated that the resonance not only requires the matching of natural frequency of field line oscillations with that of cavity oscillations but also the symmetry of their eigen functions have to be matched along the field line. The effects of finite ionospheric conductivity have been taken up recently by various authors (Yoshikawa and Itonaga, 1996; Yoshikawa et al., 1999; Nakata et al., 2000) in the simplified cartesian coordinates because of obvious complications in case of more realistic dipole geometry. Yoshikawa and Itonaga (1996) discussed the reflection and mode conversion and also examined the effect of divergent Hall current associated with the inductive electric field of fast wave on toroidal oscillations. Yoshikawa et al. (1999) numerically performed the eigenmode analysis of MHD waves including the effects of in- ductive ionosphere, an insulating atmosphere and a perfectly conducting Earth and investigated how the characteristics of field line oscillations depend on ionospheric conductivity. As they worked in straight field geometry, in their simulation scheme the Alfvén velocity is homogeneous along the field line. The dependence of eigenperiods on ionospheric conductivity was via the composite boundary conditions that defined the coupling of various regions viz. magnetosphere, ionosphere, atmosphere and solid Earth. Nakata et al. (2000) numerically depicted in straight ambient field inclined to the ionosphere in the trapezoid model as to how the transmission of magnetic perturbation depends on relative dominance of Pedersen and Hall conductivity of the ionosphere. In their simulation they found that the fundamental toroidal oscillations are governed by Pedersen conductivity whereas higher harmonics are governed by Hall conductivity. They also pointed out that the north-south asymmetry of the ground magnetic perturbations depends not only on the geocentric equatorial distance of the field lines but also on the magnetic longitude.

The first attempt to study the eigen mode excitation by any external force was made by Chen and Hasegawa (1974). In this paper they applied the Green's function technique in Cartesian geometry to study the radial structure of oscillations induced by surface eigen modes. The paper discussed only the radial variation in the magnetic field and did not address the problem of mode structure along the field line as regards their response to an impulse. Zhu and Kivelson (1989) performed the numerical analysis to study the possible existence of field line resonance and cavity modes in the plasmosphere by using box model. Walker $(1998,2000)$ provided an analytic account of excitation of cavity resonance by a disturbance in solar wind and its coupling to field line resonances in the Cartesian geometry. The papers emphasize the need for an analytic technique such as classical WKBJ method to get a physical insight of the phenomena. The most significant work in this direction came from Leonovich and Mazur (1993, 1997) where they used Green's function technique to study field line oscillations and applied the method to examine the excitation by the E-layer ionospheric currents in the dipole geometry. They used natural eigen modes for the construction of Green's function. We believe that spectral analysis will probably provide further insight into numerical results obtained by various authors and what we are attempting here is the first step towards that goal.

The statistical studies based on satellite observations (Anderson et al., 1990) revealed that the fundamental toroidal oscillations are dominant in dawn and dusk sectors and it is not that obvious in case of poloidal oscillations. The fact was then attributed to the Kelvin-Helmoltz (K-H) instability without much observational support. Still, it is believed the same and there is no concrete observational evidence supporting the issue.

In this paper we find that observed longitudinal dependence of excited oscillations can be explained in terms of response to impressed solar-wind pressure impulses at the magnetopause boundary without invoking K-H instability. In the present work a methodology using spectral representation of Green's function has been applied to compute the response of magnetospheric transverse mode oscillations to 
the time varying oscillations in current flowing at the magnetopause boundary due to sudden change in solar wind dynamic pressure. This is a simplified model for simulating the oscillations associated with the solar wind dynamic pressure. Response of the field lines is computed taking the ionosphere as perfectly reflecting. We are aware of the fact that the finite ionospheric conductivity decisively effects the periodicity and damping rate of field line oscillations although it becomes analytically difficult to handle the coupled equation in dipole field with realistic Alfvén velocity profile. In our formalism Pedersen and Hall currents can be included and will be done explicitly in future paper. We have not accounted for the radial dependence due to the propagation characteristics of the compressional mode because we have neglected the plasma response inside the magnetosphere. We are aware that compressional wave can vary radially and hence can modify the response of the field line. This we believe may change the amplitude of the excited field line oscillations but may not have an equally significant effect on the longitudinal dependence. However, there could be some modifications in the latitudinal structure. Our motivation in the paper is to see the longitudinal structure of the transient response of discrete field lines to the solar wind pressure variation in a dipole field geometry. Our efforts should be regarded as the first attempt that includes the longitudinal structure of magnetopause currents and the stated restrictions will be relaxed in future studies. The layout of the paper is as follows.

Section 2 describes the theory and analysis and how the theory could be applied to bring out the response of transverse field line oscillations (both toroidal and poloidal) to the current variations in magnetopause associated with sudden change in solar wind dynamic pressure. Highlights of the results brought out by the present investigations are stated in Section 3 and the main conclusions are stated in Section 4.

\section{Theory and Analysis}

Low frequency transverse waves in an infinitely conducting stationary, magnetized plasma with negligible plasma pressure can be described by second order wave equation (Singer et al., 1981) as

$$
\mu_{o} \rho \frac{\partial^{2}}{\partial t^{2}}\left(\xi_{\alpha} / h_{\alpha}\right)=\frac{B_{o}}{h_{\alpha}^{2}} \frac{d}{d s}\left[h_{\alpha}^{2} B_{o} \frac{d}{d s}\left(\xi_{\alpha} / h_{\alpha}\right)\right]
$$

where $\mu_{o}$ is the magnetic permeability in the vacuum, $\rho$ is the plasma mass density, $\xi_{\alpha}$ is the plasma displacement perpendicular to the field line, $s$ is the distance measured along the field line, $B_{o}$ is the ambient magnetic field, the parameter $\alpha$ signifies the mode of oscillation and determines the direction $\vec{\alpha}$ of the field line displacement and $h_{\alpha}$ is the scale factor for the normal separation between the field lines in the direction $\vec{\alpha}$ and is determined by the ambient magnetic field structure.

It should be noted that under dipole approximation

$$
h_{\alpha}=L_{d} \sin ^{3} \theta
$$

for the toroidal mode and

$$
h_{\alpha}=\frac{\sin ^{3} \theta L_{d} \sqrt{4 L_{d}-1}}{\sqrt{1+3 \cos ^{2} \theta}}
$$

for the poloidal mode, where $L_{d}$ is the geocentric distance in Earth radii of the point where the field line crosses the equator and $\theta$ is the co-latitude.

In the derivation of (1) the compression of the magnetic field has been completely neglected. If we take into account the compression then (1) gets modified as

$$
\begin{gathered}
\mu_{o} \rho \frac{\partial^{2}}{\partial t^{2}}\left(\xi_{\alpha} / h_{\alpha}\right)-\frac{B_{o}}{h_{\alpha}^{2}} \frac{d}{d s}\left[h_{\alpha}^{2} B_{o} \frac{d}{d s}\left(\xi_{\alpha} / h_{\alpha}\right)\right] \\
=-\frac{B_{o}}{h_{\alpha}^{2}} \frac{\partial b_{s}}{\partial \alpha}
\end{gathered}
$$

where, $b_{s}$ is the change in the magnetic field strength due to compression. The derivative with respect to $\alpha$ in the right hand side means that the derivative has to be taken with respect to the longitude $\phi$ for the toroidal mode and with respect to $v$ for the poloidal mode, where $(\vec{\nabla} s, \vec{\nabla} \phi, \vec{\nabla} v)$ form a right handed orthogonal system.

Any fluctuation in the magnetopause current associated with pressure change will produce a field $b_{s}$ at the field line position. It can then provide the impulse that can in turn generate a response in the field line oscillations (Lee, 1996). Thus, we can identify $b_{s}$ that appears on the right hand side with external forcing and solve for response defined by the operator on the left hand side of the equation.

We, thus try to solve equation (4) under the following assumptions: (a) The ambient magnetic field is dipolar in nature, (b) The time dependence of all the perturbed quantities is of the form $\exp [\iota \omega t]$, (c) The density distribution is given by the power law $\rho=\rho_{o}\left(r_{o} / r\right)^{m}$ (Cummings et al., 1969), where $m$ is the density index and $\rho_{o}$ is the proton mass density at $r_{o}$, the geocentric distance to the equatorial crossing point of the field line considered and $r$ is the geocentric distance to the position of interest on the field line.

According to the above assumptions equation (4) can be written in the normalized form as

$$
[L-\lambda] X(S)=f(S)
$$

where,

$$
\begin{gathered}
L=-\frac{B \sin ^{2 m} \theta}{h_{\alpha}^{2}} \frac{d}{d S}\left[h_{\alpha}^{2} B \frac{d}{d S}\right] \\
f(S)=-\frac{B \sin ^{2 m} \theta}{h_{\alpha}^{2}} \frac{\partial b_{s}^{\prime}}{\partial \alpha} \\
\lambda=\Omega^{2}
\end{gathered}
$$

The quantities are normalized as

$$
\begin{aligned}
& S=\frac{s}{R_{e}}, \Omega=\frac{\omega R_{e}}{V_{A e q}}, X=\frac{\xi_{\phi}}{R_{e} h_{\phi}}, \\
& B=\frac{B_{o}}{B_{e q}}, b_{s}^{\prime}=\frac{b_{s}}{B_{e q}}
\end{aligned}
$$

where $R_{e}$ is the Earth's radius, $V_{A e q}$ is the Alfvén velocity at the equator, $B_{e q}$ is the ambient field at the equator.

It should be noted that the function $f(S)$ in right hand side of Eq. (5) depends only on the change in the magnetic 


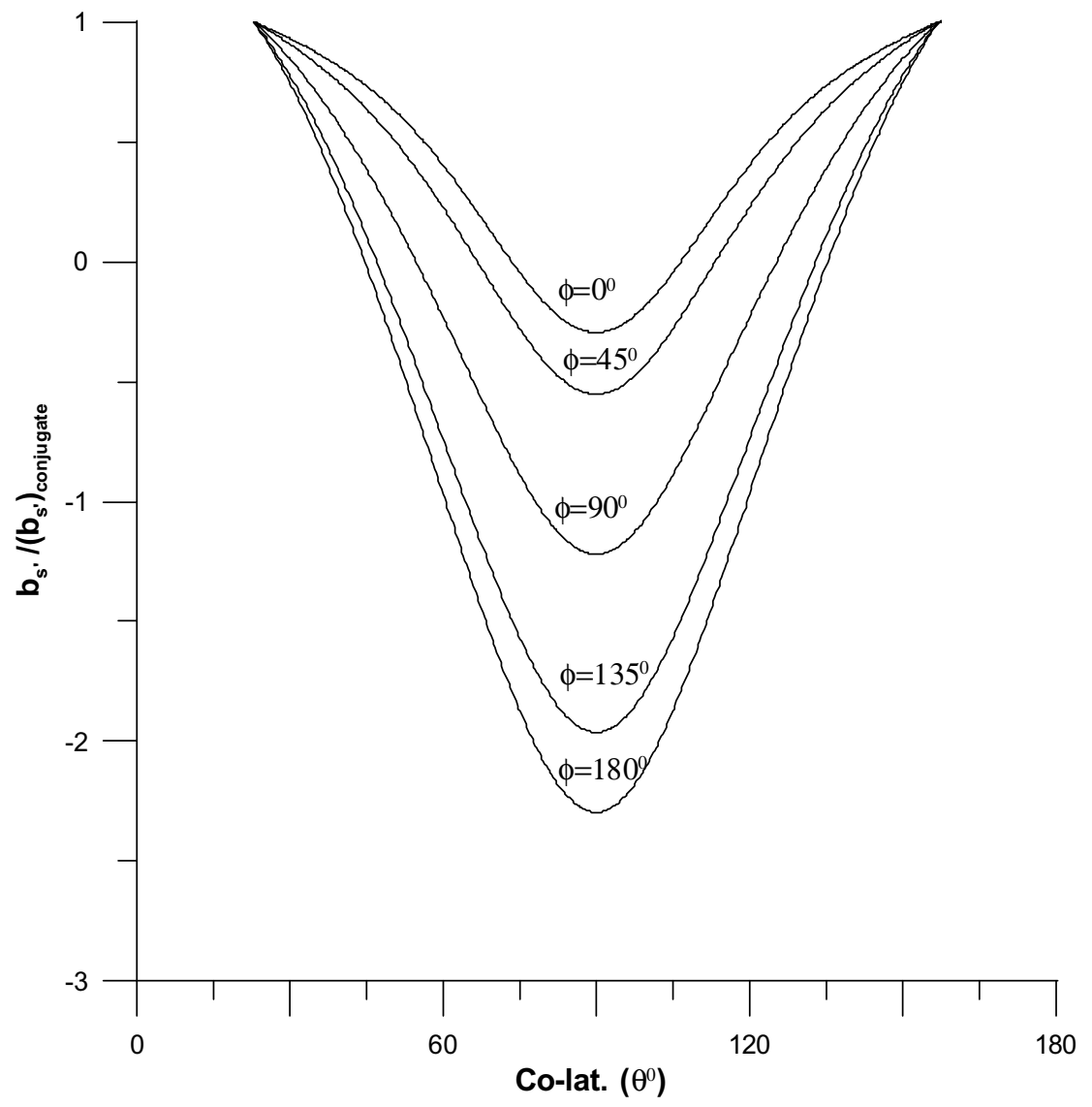

Fig. 1. The compressional component $\left(b_{s^{\prime}}=\frac{b_{s}}{B_{e q}}\right)$ of geomagnetic field variation due to the oscillations of current at the magnetopause based on Mead's model (1964) along a field line $\left(L_{d}=6.6\right)$ at different latitudes.

field due to compression caused by the change in solar wind dynamic pressure.

We proceed to solve equation (5) by spectral representation of the Green's function technique (Dudley, 1994). As a first step to apply the methodology we have to solve equation (5) with right hand side zero i.e.

$$
\left[L-\lambda_{n}\right] u_{n}(S)=0
$$

with the specified boundary conditions. It is assumed that $X(S)$ satisfies the same boundary conditions as that of $u_{n}(S)$. Assuming the ionosphere to be perfectly reflecting we analyzed the problem for the extreme cases viz. (a) ionospheric conductivity is infinitely large and (b) ionospheric conductivity is infinitesimally small. In terms of boundary conditions it amounts to saying that the eigen function $u_{n}(S)$ in equation (10) in the first case and its derivative in the second case are vanishing at the foot of the field line in the ionosphere for both the hemispheres.

Equation (10) was solved numerically for both toroidal and poloidal oscillations by using second order Runge-Kutta method and $\lambda_{n}$ was obtained by shooting method using the specified boundary conditions at the conjugate points. Typical proton density at the equator is taken to be $1 / \mathrm{cm}^{3}$ and hence the corresponding mass density $\rho_{o}$ is obtained by multiplying with the proton mass. The typical value of density index $m$ is taken to be 5 .

The typical eigen functions are symmetric (or asymmetric) about the equator for the odd (or even) harmonics for the fixed-end case (Cummings et al., 1969), while for the freeend case the even (or odd) harmonics are symmetric (or asymmetric) about the equator (Newton et al., 1978). The eigen function thus obtained is normalized in the domain and hence the normalized eigen function $u_{n}$ corresponding to a particular eigen value $\lambda_{n}$ is obtained as a function of $S$. This way the normalized solution can be obtained for any number of harmonics. The normalized eigen function will satisfy the following relation

$$
\int_{D} u_{n}^{*}(S) u_{n}(S) d S=1
$$

Then, the orthogonality of the eigen functions corresponding to different eigen values (i.e. different harmonics) is assured by applying the following criteria

$$
\int_{D} u_{m}^{*}(S) u_{n}(S) d S=0
$$

where, $m \neq n$.

Thus, the complete orthonormal set of solutions is obtained.

Before proceeding to solve equation (5) we must verify that the operator $L$ given by the equation (6) is self adjoint in the domain specified by the boundaries at the ionosphere. As the operator $L$ is real, it can easily be seen that

$$
\int_{D}(L u) v^{*} d S=\int_{D} u\left(L^{\dagger} v^{*}\right) d S
$$




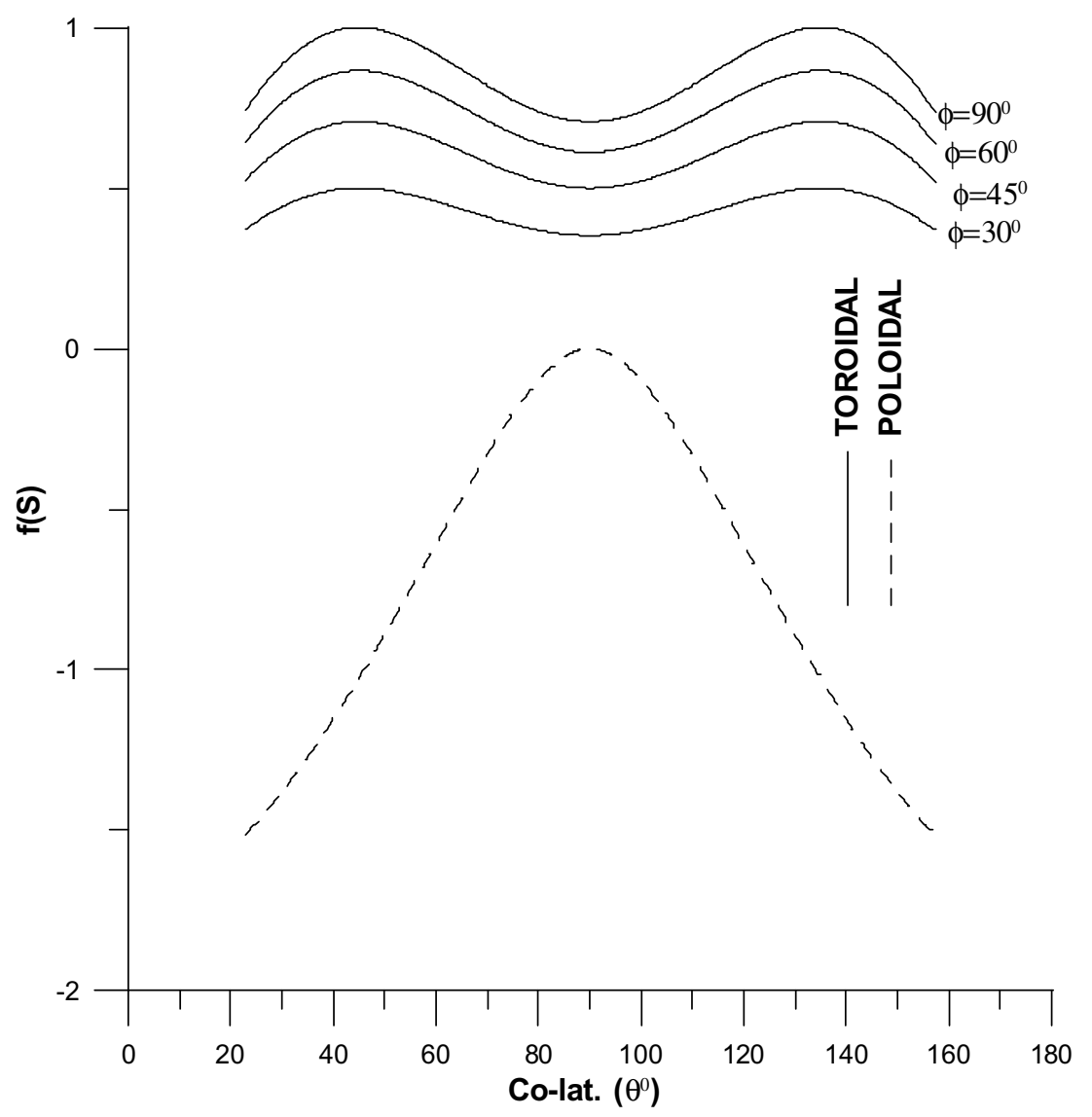

Fig. 2. The source term $f(S)$ along the field line $\left(L_{d}=6.6\right)$ for toroidal (solid curve) and poloidal (dashed curve) modes.

where, $u$ and $v$ are the solutions of equation (10) satisfying the specified boundary conditions and belong to the domain $D$ of the solution. In the present case the relation (13) holds for both types of boundary conditions.

Having confirmed that the operator $L$ is self-adjoint, a complete orthonormal set of solutions of equation (10) can be used to obtain the solution of equation (5) by constructing the Green's function $G\left(S, S^{\prime}\right)$ and the solution can be written as

$$
X(S)=\int_{D} G\left(S, S^{\prime}\right) f\left(S^{\prime}\right) d S^{\prime}
$$

where, the integration is carried out in the domain $D$ of the eigen function and $G\left(S, S^{\prime}\right)$ is given as

$$
G\left(S, S^{\prime}\right)=\sum_{n=1}^{\infty} \frac{u_{n}(S) u_{n}{ }^{*}\left(S^{\prime}\right)}{\lambda_{n}-\lambda}
$$

Here, the summation runs over the harmonics.

Finally, the solution of equation (5) can be written by combining equations (14) and (15) as

$$
X(S)=\sum_{n=1}^{\infty} \frac{u_{n}(S)}{\lambda_{n}-\lambda} \int_{D} u_{n}{ }^{*}\left(S^{\prime}\right) f\left(S^{\prime}\right) d S^{\prime}
$$

The integral within the summation sign in the right hand side of equation (16) generates the coefficients of $u_{n}$ in the expansion of $f\left(S^{\prime}\right)$ in terms of the eigenmodes. Response $X(S)$ of a given mode depends on how much the mode contributes in the expansion of the forcing term and also how close $\lambda$ is to $\lambda_{n}$. The response maximizes when the frequency of the wave is in the vicinity of one of the natural frequencies of field line oscillations and $u_{n}$ closely reproduces the latitudinal structure of the function $f(S)$.

For numerical computation, we impart a small imaginary part $\gamma$ to the wave frequency $\omega$ in order to avoid singularity at the resonant condition i.e. when $\lambda=\lambda_{n}$. If we write $\omega=\omega_{r}+\iota \gamma$ then in resonant condition it can be seen that $\left|\lambda_{n}-\lambda\right| \sim 2 \Gamma \Omega_{r}, \Omega_{r}$ and $\Gamma$ are the normalized $\omega_{r}$ and $\gamma$ and the normalization factor is same as that for $\omega$ as given in equation (9). Thus, greater response are expected for the lower harmonics. For the present analysis the value of $\gamma$ is chosen to be 0.005 . The ratio of $\gamma$ to the fundamental normal mode angular frequency is of the order of $10^{-4}$ and the ratio will still be smaller for higher mode frequencies. Though the response should be less for higher $\gamma$, it is not important for the present analysis as we are interested in relative magnitude of response for different wave periods. Here, a finite $\gamma$ has been taken just to avoid singularity and to get physically meaningful response.

One of the important problems in determining the response of field line oscillations is that of specifying the exact representation of the impulse term. It is obvious from the integral in equation (14) that the magnitude of response of the field line oscillations is sensitive to the latitudinal structure of the impulse term. To obtain a preliminary estimate of the induced field line oscillations, it is necessary to use a working model that at least qualitatively represents the lat- 


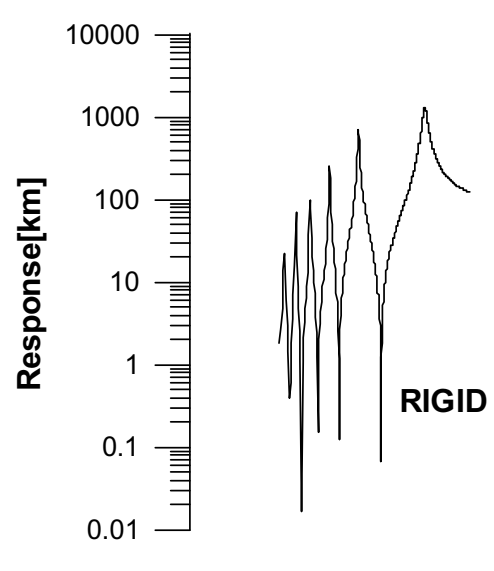

POLOIDAL
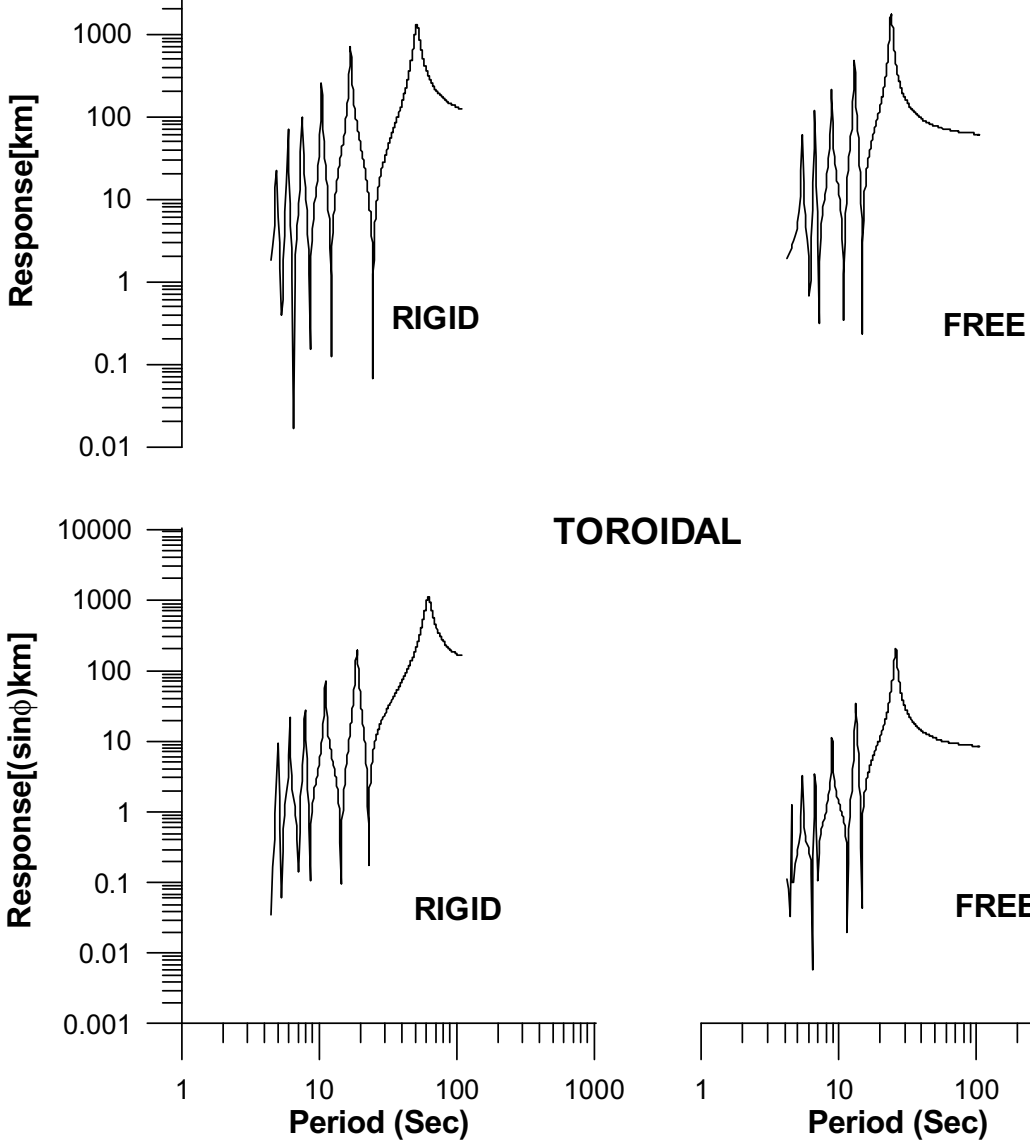

FREE

TOROIDAL

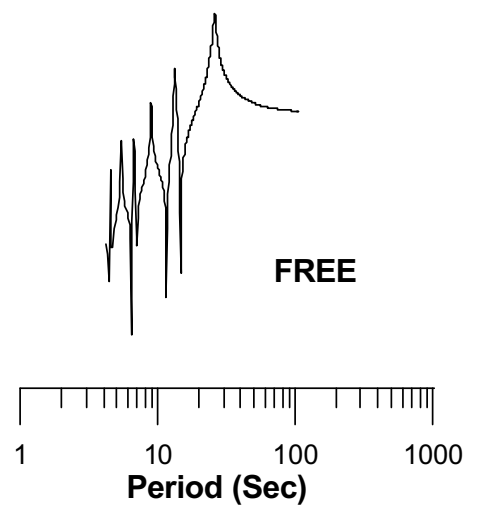

Fig. 3. The equatorial response of a particular magnetic shell $\left(L_{d}=6.6\right)$ as a function of wave-periods. The upper and lower panels respectively represent poloidal and toroidal modes, whereas left and right panels represent rigid-end case and free-end case respectively. Peaks in decreasing sequence of wave-periods for rigid case correspond to odd harmonics i.e. harmonic numbers $1,3,5 \ldots$ and those in case of free-end correspond to even harmonics i.e. harmonic numbers $2,4,6 \ldots$ The toroidal response at a given longitude can be obtained in kilometers by multiplying the ordinate with a factor $\sin \phi$.

itudinal structure of the impulse term. Given the distribution of magnetopause currents associated with the impulse term, one can, in principle estimate the structure of magnetic impulse along any given field line. The model of Mead (1964) provides one such estimate. The model neglects the interplanetary magnetic field and also the plasma-pressure effects within the magnetosphere. The model also assumes that the Earth's dipole is normal to the direction of the undeflected solar wind. According to the model the components of magnetic field in polar coordinate due to surface current at a given magnetopause stand-off distance are given as

$$
\begin{aligned}
B_{r}= & -2 B_{o}\left(\frac{R_{e}}{r}\right)^{3} \cos \theta+B_{1}\left(\frac{R_{e}}{a}\right)^{3} \cos \theta \\
& -2 B_{2}\left(\frac{R_{e}}{a}\right)^{4}\left(\frac{r}{R_{e}}\right) \cos \theta \sin \theta \cos \phi \\
B_{\theta}= & -B_{o}\left(\frac{R_{e}}{r}\right)^{3} \sin \theta-B_{1}\left(\frac{R_{e}}{a}\right)^{3} \sin \theta \\
& +B_{2}\left(\frac{R_{e}}{a}\right)^{4}\left(\frac{r}{R_{e}}\right)\left(2 \sin ^{2} \theta-1\right) \cos \phi
\end{aligned}
$$

$$
B_{\phi}=B_{2}\left(\frac{R_{e}}{a}\right)^{4}\left(\frac{r}{R_{e}}\right) \cos \theta \sin \phi
$$

where, $B_{o} \sim 0.311$ Gauss, $B_{1} \sim 0.25$ Gauss, $B_{2} \sim$ 0.21 Gauss, $a$ is the equatorial stand-off distance from the point dipole to the magnetopause in the noon meridian. It is obvious that the first term in expressions (17) and (18) are the dipolar part of the geomagnetic field components.

With a little bit of algebra it can be seen that the compressional component of geomagnetic field variation due to the oscillations of currents at the magnetopause based on Mead's model is given as

$$
\begin{array}{r}
b_{s}=\frac{1}{\sqrt{1+3 \cos ^{2} \theta}}\left[-3 \frac{B_{1}}{R_{e}}\left(\frac{R_{e}}{a}\right)^{4}\left(1-3 \cos ^{2} \theta\right)+\right. \\
\left.4 \frac{B_{2} L_{d} \sin ^{3} \theta \cos \phi}{R_{e}}\left(\frac{R_{e}}{a}\right)^{5}\left(1+2 \cos ^{2} \theta\right)\right] \Delta a
\end{array}
$$

where, $\Delta a$ is the displacement of the magnetopause in the direction perpendicular to its surface. The variation of $b_{s}$ along a particular field line $\left(L_{d}=6.6\right)$ has been shown in Fig. 1. 


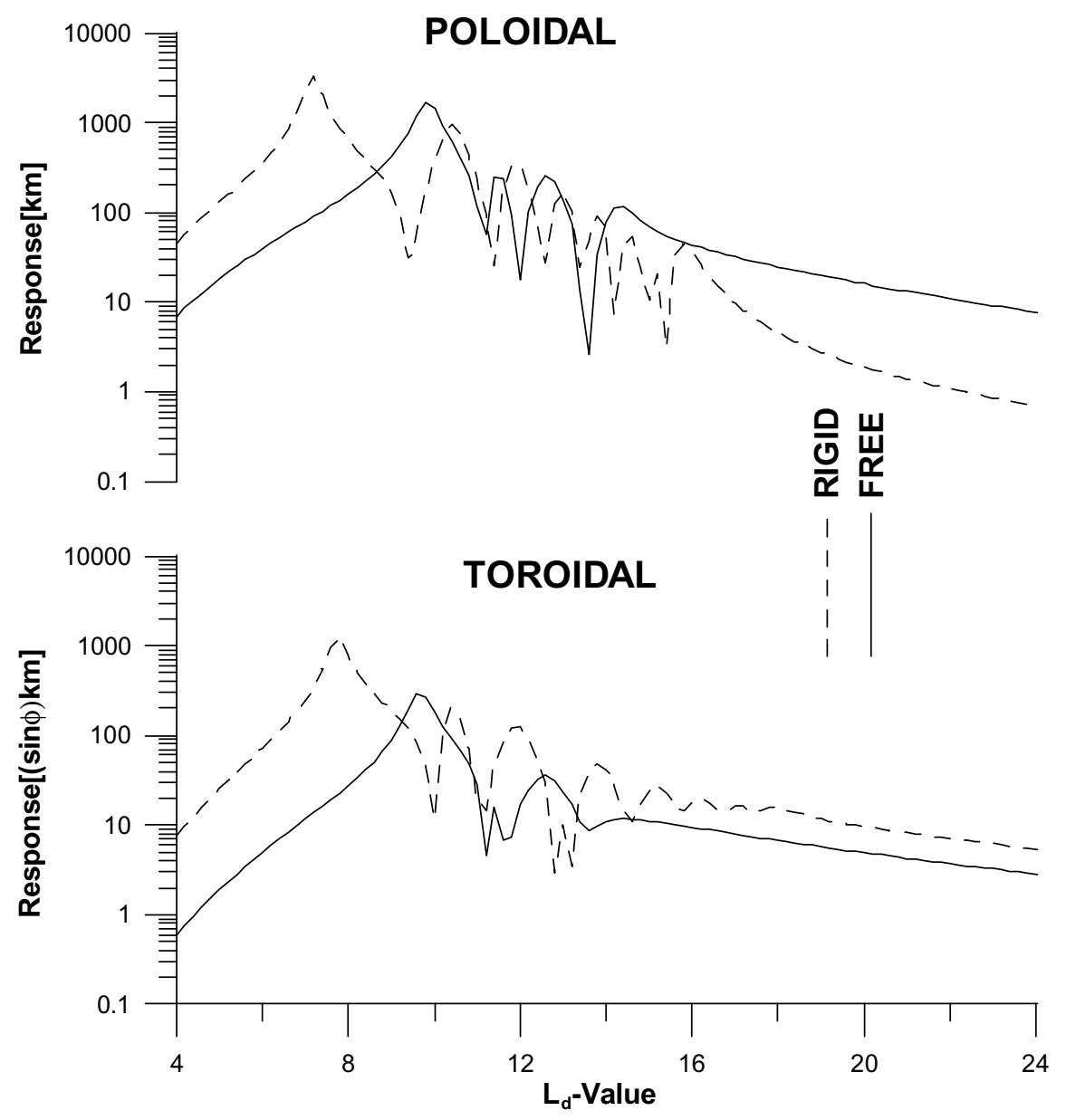

Fig. 4. The equatorial response of different magnetic shells characterized by their $L_{d}$ values for a fixed source-period (120 Sec). The upper and lower panels represent the poloidal and toroidal oscillations respectively. The curve in dashed line is for rigid boundary case and the curve in solid line is for free boundary case. The response at a given longitude for toroidal oscillations can be obtained in kilometers by multiplying the ordinate with a factor $\sin \phi$.

If we take the time variation of $\Delta a$ as $\exp (\iota \omega t)$ then $f(S)$ in the normalized equation (5) for toroidal mode is of the form

$$
f(S)=\frac{4 B B_{2}^{\prime} \sin \phi \sin ^{2 m} \theta\left(1+2 \cos ^{2} \theta\right)}{L_{d} \sin ^{3} \theta a^{\prime 5} \sqrt{1+3 \cos ^{2} \theta}} \Delta a^{\prime}
$$

and for the poloidal mode $f(S)$ in its dominant form can be written as

$$
f(S)=-\frac{54 B B_{1}^{\prime} \cos ^{2} \theta\left(1+\cos ^{2} \theta\right) \sin ^{2 m} \theta}{h_{v} L_{d} a^{\prime 4} \sin \theta\left(1+3 \cos ^{2} \theta\right)^{2}} \Delta a^{\prime}
$$

where,

$$
B_{1}^{\prime}=\frac{B_{1}}{B_{e q}}, B_{2}^{\prime}=\frac{B_{2}}{B_{e q}}, a^{\prime}=\frac{a}{R_{e}}, \Delta a^{\prime}=\frac{\Delta a}{R_{e}}
$$

In expressing $f(S)$ for poloidal mode in equation (22) the term depending on $\cos \phi$ is of one order smaller and hence has been neglected.

From equations (21) and (22) it is evident that the function $f(S)$ is symmetric about the equator for both the toroidal and poloidal oscillations. The variation of $f(S)$ along a particular field line $\left(L_{d}=6.6\right)$ has been shown in Fig. 2 for both toroidal and poloidal oscillations. The longitudinal variation of $f(S)$ in case of toroidal oscillations is to be noted. It follows straight away that only symmetric modes will respond to the forcing. Note that in the case of fixed-end the odd harmonics are symmetric and in the case of free-end the even harmonics are symmetric. Also, it can be seen that the $f(S)$ will depend upon the amplitude of the magnetopause displacement $\Delta a^{\prime}$ and the stand-off distance $a^{\prime}$ of the magnetopause. In the present analysis we have taken the value of $\Delta a^{\prime}$ as 0.1 and that of $a^{\prime}$ as 10 . Moreover, $f(S)$ should be different for different magnetic shells characterized by their $L_{d}$ values. In fact $f(S)$ decreases with increase in $L_{d}$. Note that $f(S)$ is longitude dependent in case of toroidal mode and axially symmetric in case of the poloidal mode. The presence of $\sin \phi$ in the expression of $f(S)$ for toroidal mode will produce a longitudinal variation in the response. The presence of $a^{\prime}$ in the denominator with one power less and the factor $\cos ^{2} \theta$ in the numerator in the expression of $f(S)$ for the poloidal mode as compared to that of toroidal mode indicate the greater response for poloidal mode as compared to that for toroidal mode. All these characteristics should be reflected in the response $X(S)$ of magnetic field lines.

Constructing the Green's function using the normal mode solutions of field line oscillations by equation (15) and plugging it along with $f(S)$ in equation (14), solution $X(S)$ of 

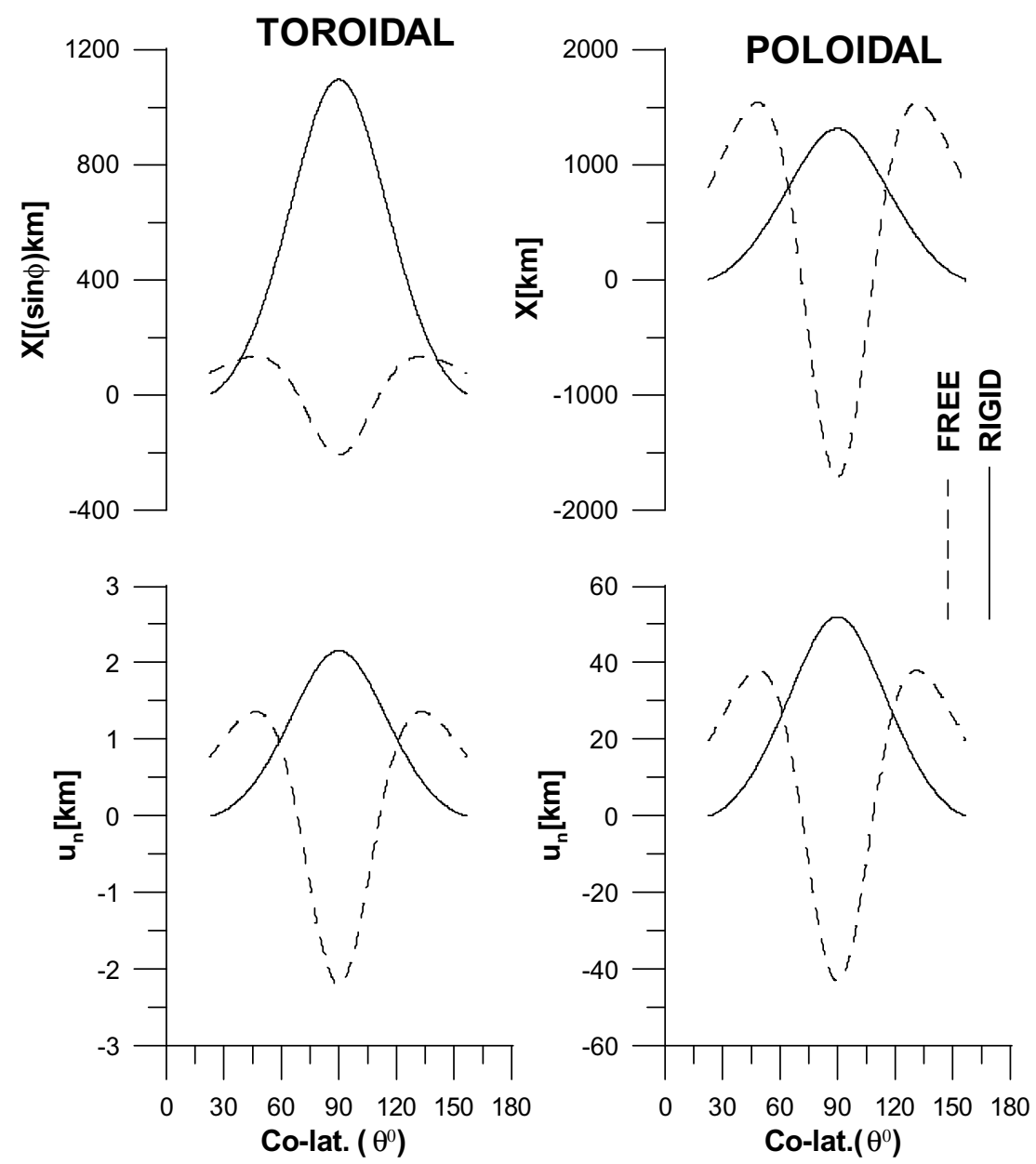

Fig. 5. The latitudinal response-structures of dominant modes (fundamental for rigid-end case and second harmonic for free-end case) along a field line $\left(L_{d}=6.6\right)$. The left and right panels represent toroidal and poloidal oscillations respectively, whereas dashed and solid curves respectively represent free and rigid-end cases. The lower panel shows the mode structure when there is no compression whereas the upper panel represents the field line structure in presence of compression.

equation (5) could be obtained. In other words, the response of toroidal and poloidal oscillations to the oscillations of current at the magnetopause associated with the solar wind dynamic pressure is computed using Mead's model in perfectly reflecting ionospheric conditions. The response were computed for fixed $L_{d}$ taking different periods of driving oscillations (i.e. for different corresponding $\lambda=\Omega^{2}$ ) for both types of boundary conditions (i.e. 'rigid-end' and 'freeend') (Fig. 3). Also, we have examined as to how different magnetic shells respond to a particular driving frequency (Fig. 4). The choice of driving period is based on Sibeck et al. (1989) and is taken as $120 \mathrm{~s}$ periodicity which corresponds to azimuthal oscillations due to solar wind dynamic pressure variation. The field line structures of response for dominant modes (i.e. fundamental in case of rigid-end and second harmonic in case of free-end) have also been computed taking $L_{d}=6.6$ for both the toroidal and poloidal oscillations (Fig. 5). Longitudinal variation of response have been computed for the first time for both rigid (Fig. 6) and free-end (Fig. 7) for various driving periods only in case of toroidal oscillations as such longitude dependence is not that obvious for poloidal oscillations. This could be possible because we took the three dimensional distribution of compres- sional source. It is not possible to get the longitudinal variation of response in the simple box geometry. The results and their various implications have been discussed in the following section.

\section{Results and Discussions}

The analysis shows that for a particular magnetic shell characterized by its $L_{d}$-value responds to different periods of oscillation of the magnetopause due to the variation in solar wind dynamic pressure as depicted in Fig. 3. It should be noted that the term 'response' in the present context means the displacement of the field lines due to the external forcing i.e. due to the change in solar wind dynamic pressure. The response of toroidal and poloidal modes have been computed to different periods of driving force at the geostationary orbit ( $L_{d}=6.6$ ) for both the cases of perfectly reflecting ionosphere.

Also, the response of toroidal and poloidal modes at different magnetic shells characterized by different $L_{d}$ values to a given period of driving force (120 sec. in the present case) have been computed for both the cases of perfectly reflecting ionosphere as depicted by Fig. 4.

In case of toroidal modes (refer to lower panels of Figs. 3 


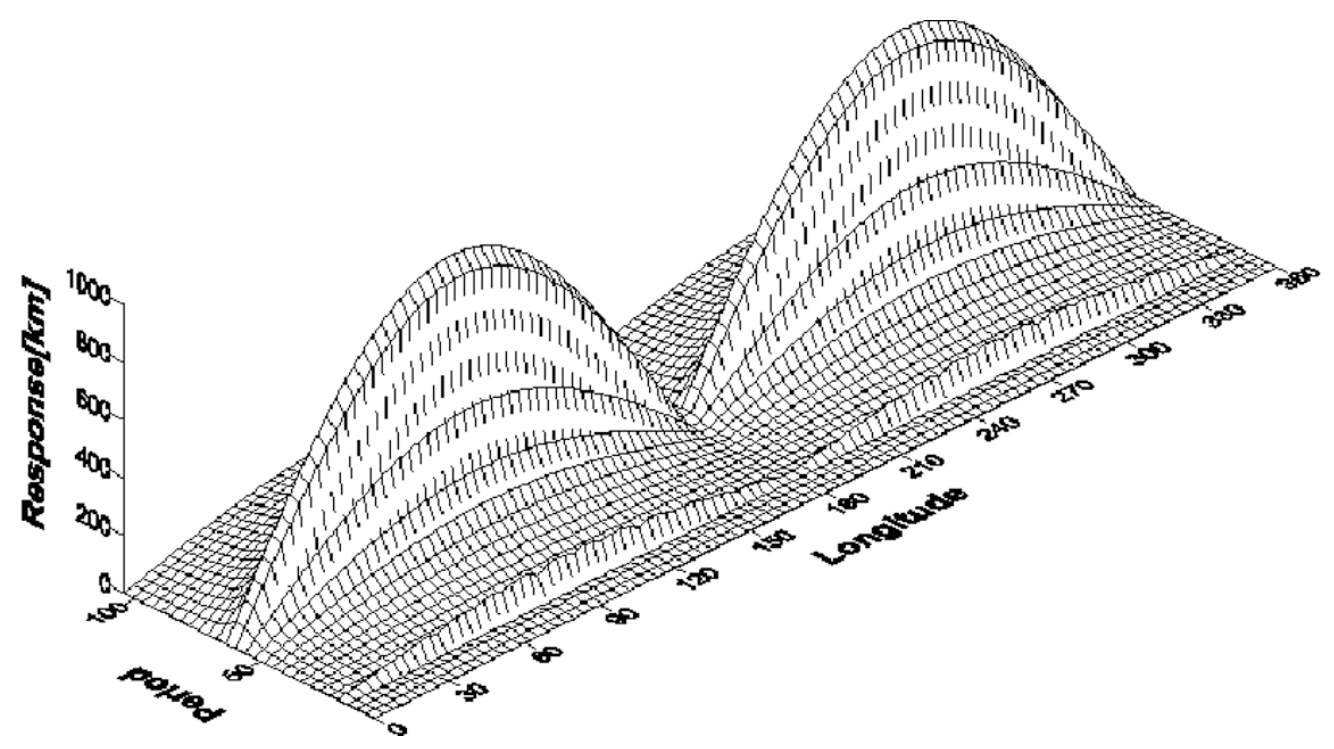

Fig. 6. 3-D plot showing the response variation with wave-period and the longitude for toroidal oscillation in rigid-end case. Dotted lines represent response variation at constant longitude and solid lines show response variation at constant period.

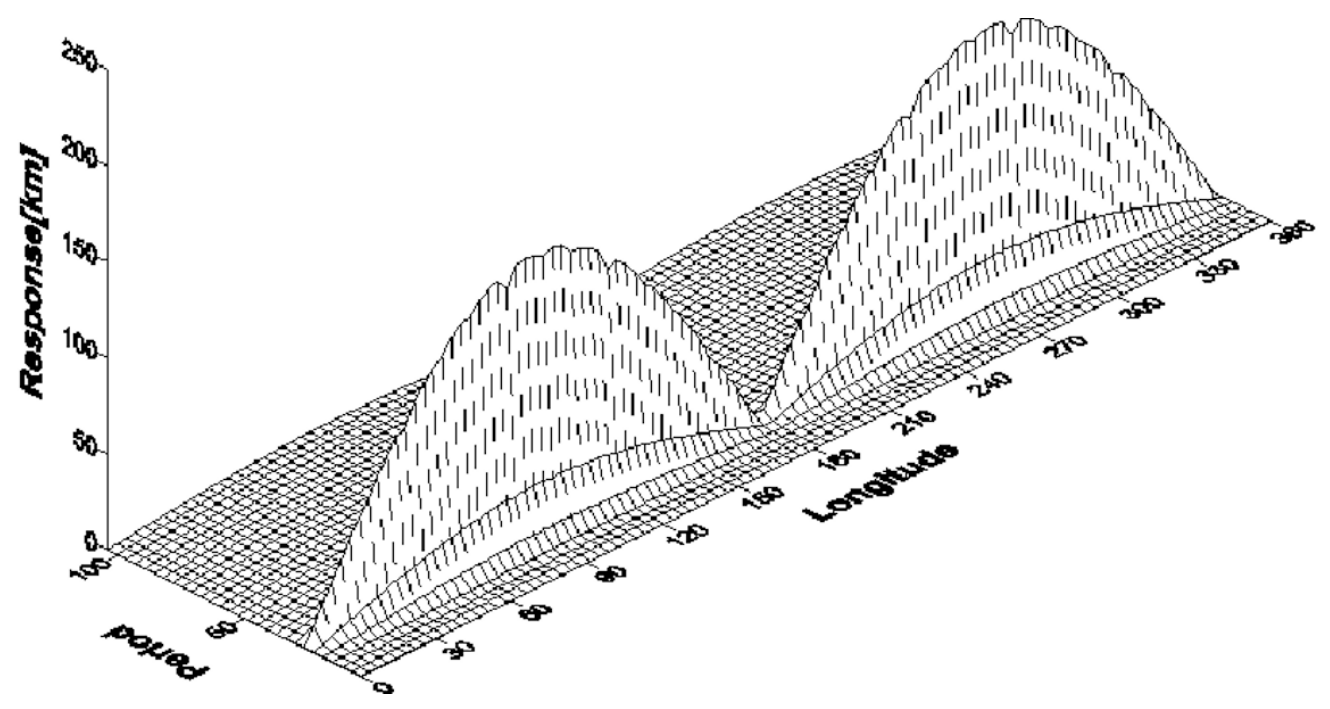

Fig. 7. 3-D plot showing the response variation with wave-period and the longitude for toroidal oscillation in free-end case. Dotted lines represent response variation at constant longitude and solid lines show response variation at constant period.

and 4) a multiplicative factor $\sin \phi$ is of paramount importance and appears only in case of toroidal mode response. A statistical studies of pulsations observed by AMPTE/CCE reveals that fundamental toroidal modes predominantly occur in the dawn and the dusk sector and supposedly driven by K-H instability (Anderson et al., 1990). Our analysis shows that response to pressure oscillations will also generate enhanced toroidal oscillations in the dawn/dusk sector through $\sin \phi$ term. The dominance of poloidal oscillations in dawn and dusk sectors are not obvious in our analysis which is consistent with the observations of Anderson et al. (1990). The longitudinal variation of toroidal response for various driving periods have been computed and shown in Fig. 6 (rigid-end case) and Fig. 7 (free-end case).

We have presented the response at the equator at different driving periods in Fig. 3 as the features of harmonics of field line oscillations are known at the equatorial latitude and thus will provide a better understanding of the phenomena. We have also computed the response all along the field line for dominant modes (Fig. 5) viz. the fundamental for rigid-end case and second harmonic for free-end case. Main features of the results obtained through the present analysis can be stated as follows:

(a) The positions of peaks in Fig. 1 match with the timeperiods of harmonics of natural modes of field line oscillations as given by Cummings et al. (1969). Peaks in rigid-end case correspond to the odd harmonics (refer to left panel of Fig. 3) and peaks in free-end case correspond to the even harmonics (refer to right panel of Fig. 3).

The above results suggest that the waves caused by external forcing at the magnetopause boundary may excite the field line oscillations through resonance as revealed in the 
numerical model of Lee (1996).

(b) For a given magnetic shell, poloidal mode shows larger response as compared to toroidal.

This conclusion can be drawn by comparing the rigidend response of toroidal mode with rigid-end response of poloidal mode and free-end response of toroidal mode with free-end response of poloidal mode (Fig. 3). The reason lies in the model of the source term $f(S)$. The ratio of the amplitudes of the source term for the poloidal (Eq. (22)) to the toroidal (Eq. (21)) modes at the equator (for which the result has been presented) turns out to be much greater than one. This shows that the response of the field line depends on the amplitudes of the source term and is larger for the larger amplitudes.

(c) It is revealed through the analysis that odd harmonics are excited in case of 'rigid-end' boundary and even harmonics are excited in case of 'free-end' boundary (Fig. 3) as expected from expression given in equation (14). Thus, it is predominantly fundamental mode that responds in the 'rigid-end' case and second harmonic that responds in the 'free-end' case.

(d) For a given periodicity (120 sec. in the present case) it is seen that different magnetic shells respond at different harmonics (Fig. 4).

For 'rigid-end' boundary the dominant response is due to fundamental at lowest $L_{d}$ value 7.8 and 7.2 for toroidal and poloidal modes respectively (dashed curves in Fig. 4).

In case of 'free-end' boundary the dominant response is at $L_{d} \sim 9.8$ for toroidal and poloidal both as shown by solid curves in Fig. 4 and is due to second harmonic.

It is seen in case of higher $L_{d}$ shells, the responses, as expected, go down as they are attributed to higher harmonics.

It is seen that the position of peaks are significantly different only in case of fundamental for toroidal and poloidal modes whereas they almost coincide for other harmonics. This is because of the fact that the normal mode frequencies of toroidal and poloidal modes differ significantly only in case of fundamental and the difference reduces to less than $2 \%$ for the second harmonic and the frequencies almost coincide for higher modes. That is why for a fixed period the second harmonic are excited approximately on the same magnetic shell for toroidal and poloidal modes as stated in the previous point.

The responses at higher values of $L_{d}$ are attributed to higher harmonics. The decrease in response with increasing $L_{d}$ should be noted.

Further, it can be seen that for a given driving frequency the response of the dominant mode of 'free-end' case is much smaller than that of 'rigid-end' case. Physically, it is quite understandable that for a given driving period the dominant 'free-end' oscillation takes place at higher $L$-shell and hence should show off a lesser response.

The analysis reveals that even for the small amplitude of displacement at the magnetopause boundary $\left(0.1 R_{e} \sim 637\right.$ $\mathrm{km})$ the dominant response at the resonant magnetic shell could be of the order of $1000 \mathrm{~km}$.

(e) The field line structures of the dominant modes (viz. fundamental in rigid-end case and second harmonic in freeend case) have been computed and presented in Fig. 5. The solid curve represents the fundamental for rigid-end case and the dashed curve represents the second harmonic in free-end case. The lower panel represent the normal modes without taking into account the compression at the magnetopause boundary and the upper panel shows the response of the field line in presence of the compression.

The enhancement of the field line displacement because of the resonance caused by compression at the magnetopause boundary is clearly brought out by the analysis. The displacement gets enhanced almost by the order of three. The factor $\sin \phi$ in case of toroidal oscillations clearly indicates the longitudinal dependence of the mode and arises due to the three dimensional model of the compression at the magnetopause boundary.

(f) the longitudinal variations of toroidal response have been computed for various driving periods for both rigid-end (Fig. 6) and free-end (Fig. 7) cases. The dashed curve shows the response variations at the given longitude whereas solid curve shows the variations for given driving period.

The most significant result of the present analysis is the dominance of toroidal mode in dawn/dusk sector that is brought out by taking the three dimensional model of compresional wave caused by solar wind dynamic pressure variation at the magnetopause boundary. This feature is clearly reflected in our analysis as can be seen from Figs. 6 and 7. The result is consistent with the observational findings of Anderson et al. (1990).

It is traditionally believed that the dominant nature of observed toroidal oscillation in the dawn and the dusk sectors can be attributed to K-H instability, but without much observational evidences. The present analysis does not account for the propagation aspect (and hence the shear in velocity) of the compressional waves generated by the solar wind pressure variation at the magnetopause boundary and still could reveal the dominance of toroidal oscillations in the dawn and the dusk regions. We do not straight away reject the K-H instability hypothesis and leave it to the future observations, though our analysis sees the phenomena as a direct consequence of pressure variation at the magnetopause boundary. Theoretically it will not be possible to get this result if the source term is assumed only to be latitude dependent.

The above results give the qualitative description of how the field line oscillations could be excited due to sudden change in solar wind dynamic pressure. The peak positions in Fig. 3 will change if we make changes in density index $m$ and $L_{d}$ as normal mode frequencies critically depend on these parameters. Also, in Fig. 4 peaks will occur at different magnetic shells if we change the wave-period. The provisions for making the changes in the parameters has been made in the algorithm, though the results presented here are for the particular values of these input parameters.

If we increase the damping factor $\gamma$, the magnitude of the response will decrease, but their relative magnitude of different harmonics will remain unaffected. This could be misleading as we have not introduced the damping selfconsistently. This could be possible by imparting the finite conductivity to the ionosphere and solving the couple equations that will generate the field line resonance via cavity oscillations self-consistently. In our analysis we use Green's function for computing response. To be precise the normal modes used for the construction of Green's function should 
be computed self-consistently by solving coupled equations in realistic field geometry and for realistic Alfvén wave profile and realistic ionospheric conditions. Recent works on eigen value of field line oscillations have been attempted by taking into account the Padersen and Hall conductivity (Yoshikawa and Itonaga, 1996; Yoshikawa et al., 1999; Nakata et al., 2000), but in simplified Cartesian geometry. We have worked in two extreme ionospheric conditions in the dipole field geometry taking a general density profile (and hence the Alfvén velocity profile) because the problem becomes complex for Green's function construction for finite ionospheric conductivity. Here, we have introduced the damping factor $\gamma$ on purely mathematical basis to avoid singularity otherwise the expression for $G\left(S, S^{\prime}\right)$ and $X(S)$ will blow up as is evident from equations (15) and (16) respectively. In our formalism provisions can be made for inclusions of Pedersen and Hall currents and will be explicitly done in future paper.

\section{Conclusions}

In order to estimate the field due to surface current at the magnetopause for a given magnetopause stand-off distance, we have taken a simplified model for solar wind dynamic pressure due to Mead (1964) which does not account for the propagation of the wave from the magnetopause to the magnetic shells. In real life situation velocity of propagation and hence the time of arrival will be altered significantly. This is not a serious limitation as far as the motivation of the present work is concerned. The magnitude of the impulse is also expected to be reduced. But at least as a first estimate, it is probably worthwhile working with the simple model while tacitly assuming that the latitudinal structure of the computed response will not be seriously altered. We have taken cognizance of the mode structure along the field line as well as the longitudinal structure of the impulse forcing. However, response of the field line oscillations have been computed neglecting current setup in the magnetosphere as a part of the propagation of the impulse from the boundary to the point of resonance. This is a major shortcoming that has to be overcome in future efforts. Nevertheless, the analysis clearly demonstrates that the field line resonances, as a mechanism of observed geomagnetic pulsations, could be driven by the sudden change in solar wind dynamic pressure. Based on the excellent correlation between pulsations observed on the ground and the solar wind dynamic pressure variation Farrugia et al. (1989) had suggested that the sudden change in the solar wind dynamic pressure could be responsible for driving these long period oscillations. Even though we have worked with simple model of Mead (1964), this aspect has clearly been brought out through our analysis. Also, the dominance of fundamental mode of toroidal oscillations in dawn/dusk sectors as revealed by satellite observations (Anderson et al., 1990) is brought out very naturally through our analysis. Though the K-H instability may be one of the candidates for driving such oscillations, the dominance of toroidal oscillations in dawn/dusk sector also appears as a direct consequence of three dimensional structures of magnetopause currents caused by sudden change in solar wind dynamic pressure.

Parallel developments (Southwood and Kivelson, 1990;
Chen and Hasegawa, 1974) have considered the radial propagation effect in simplified geomagnetic field geometry, thus ignoring the realistic mode structure along the field line and the latitudinal structure of the impulse forcing. The two approaches have to converge in future. We hope to incorporate it through an iterative algorithm.

We also want to make a point about the methodology applied to solve the problem through spectral representation of Green's function that the method has immense potential in the sense that response to the coupling of complex forcing associated with $\mathrm{K}-\mathrm{H}$ instability or tearing instability can also be evaluated given $b_{s}$ the compression associated with the driving force.

Acknowledgments. The editor thanks D.-H. Lee and another referee for their assistance in evaluating this paper. T. Iyemori, Editor in charge

\section{References}

Allan, W., S. P. White, and E. M. Poulter, Magnetospheric coupling of hydromagnetic waves - initial results, Geophys. Res. Let., 12, 287, 1985.

Allan, W., E. M. Poulter, and S.P. White, Magnetospheric wave coupling in the magnetosphere-plasmapause effects on impulse-excited resonances, Planet. Sp. Sci., 34, 1189, 1986.

Anderson, B. J., M. J. Engebretson, S. P. Rounds, L. J. Zanetti, and T. A. Potemra, A statistical study of Pc3-5 pulsations observed by the AMPTE/CCE magnetic fields experiment 1. Occurrence distributions, J. Geophys. Res., 95, 10,495, 1990.

Baumjohann, W., H. Junginger, G. Haerendel, and O. H. Bauer, Resonant Alfvén waves excited by a sudden impulse, J. Geophys. Res., 89, 2765, 1984.

Chen, L. and A. Hasegawa, A theory of long-period magnetic pulsations2. impulse excitation of surface eigenmode, J. Geophys. Res., 79, 1033, 1974.

Cummings, W. D., R. J. O'Sullivan, and P. J. Coleman, Standing Alfvén waves in the magnetosphere, J. Geophys. Res., 74, 778, 1969.

Dudley, D. G., Mathematical Foundations for Electromagnetic Theory, 99 pp., Institute of Electrical and Electronics Engineers, Inc., Newyork, 1994.

Dungey, J. W., Electrodynamics of the outer atmospheres, Rep., 69, Ions. Res. Lab. Pa. State Univ., University Park, 1954.

Farrugia, C. J., M. P. Freeman, S. W. H. Cowley, D. J. Southwood, M. Lockwood, and A. Etemadi, Planet. Sp. Sci., 37, 589, 1989.

Fujita, S. and V. L. Patel, Eigenmode analysis of coupled magnetohydrodynamic oscillations in the magnetosphere, J. Geophys. Res., 97, 13,771, 1992.

Fujita, S., M. Itonaga, and H. Nakata, Relationship between the Pi2 pulsations and the localized impulsive current associated with the current disruption in the magnetosphere, Earth Planets Space, 52, 267, 2000.

Fujita, S., T. Mizuta, M. Itonaga, A. Yoshikawa, and H. Nakata, Propagation properties of transient MHD impulses in the magnetosphere-ionosphere system: The 2D model of the Pi2 pulsations, Geophys. Res. Let., 28, 2161, 2001.

Itonaga, M., A. Yoshikawa, and K. Yumoto, One-dimensional transient response of the inner magnetosphere at the magnetic equator, 2. Analysis of waveform, J. Geomag. Geoelectr., 49, 49, 1997.

Lee, D.-H., Dynamics of MHD wave propagation in the low-latitude magnetosphere, J. Geophys. Res., 101, 15,371, 1996.

Leonovich, A. S. and V. A. Mazur, Theory of transverse small-scale standing Alfvén waves in an axially symmetric magnetosphere, Planet. Sp. Sci., 41, 697, 1993.

Leonovich, A. S. and V. A. Mazur, A model equation for monochromatic standing Alfvén waves in the axially symmetric magnetosphere, J. Geophys. Res., 102, 11,443, 1997.

Mead, G. D., Deformation of the geomagnetic field by the solar wind, $J$. Geophys. Res., 69, 1181, 1964.

Nagata, T., S. Kokubun, and T. Ijima, Geomagnetically conjugate relationships of giant pulsations at Syowa Base, Antarctica, and Reykjavik, Iceland, J. Geophys. Res., 68, 4621, 1963.

Nakata, H., S. Fujita, A. Yoshikawa, M. Itonaga, and K. Yumoto, Ground magnetic perturbations associated with the standing toroidal mode oscil- 
lations in the magnetosphere-ionosphere system, Earth Planets Space, 52, 601, 2000.

Newton, R. S., D. J. Southwood, and W. J. Hughes, Damping of geomagnetic pulsations by the ionosphere, Planet. Sp. Sci., 26, 201, 1978.

Schulz, M. and L. J. Lanzerotti, Particle diffusion in radiation belts, Springer-Verlag, Berlin Heidelberg Newyork, 1974.

Sibeck, D. G., W. Baumjohann, and R. E. Lopez, Solar wind dynamic pressure variations and transient magnetospheric signatures, Geophys. Res. Let., 16, 13, 1989.

Singer, H. J., D. J. Southwood, R. J. Walker, and M. G. Kivelson, Alfvén wave resonances in a realistic magnetospheric magnetic field geometry, J. Geophys. Res., 86, 4589, 1981

Sinha, A. K. and R. Rajaram, An analytic approach to toroidal eigen mode, J. Geophys. Res., 102, 17,649, 1997.

Southwood, D. J. and W. J. Hughes, Theory of hydromagnetic waves in the magnetosphere, Space Sci. Rev., 35, 301, 1983.

Southwood, D. J. and M. G. Kivelson, The hydromagnetic response of the magnetospheric cavity to changes in solar wind pressure, J. Geophys. Res., 95, 2301, 1990 .
Sugiura, M., Evidence of low frequency hydromagnetic waves in the exosphere, J. Geophys. Res., 66, 4087, 1961.

Walker, A. D. M., Excitation of magnetohydrodynamic cavities in the magnetosphere, J. Atmos. Ter. Phys., 60, 1279, 1998.

Walker, A. D. M., Coupling between waveguide modes and field line resonances, J. Atmos. Ter. Phys., 62, 799, 2000.

Yoshikawa, A. and M. Itonaga, Reflection of shear Alfvén waves at the ionosphere and the divergent Hall current, Geophys. Res. Let., 23, 101 1996.

Yoshikawa, A., M. Itonaga, S. Fujita, H. Nakata, and K. Yumoto, Eigenmode analysis of field line oscillations interacting with the ionosphereatmosphere-solid earth electromagnetic coupled system, J. Geophys. Res., 104, 28,437, 1999.

Zhu, X. M. and M. G. Kivelson, Global mode ULF oscillations in a magnetosphere with a monochromatic Alfvén velocity profile, J. Geophys. Res., 94, 1479, 1989.

A. K. Sinha (e-mail: ashwini@iig.iigm.res.in) and R. Rajaram 